\title{
35 Jahre Sparpolitik im Gesundheitswesen
}

\section{Peter Streckeisen}

Soziologe, PD Dr. phil., Dozent am Institut für Vielfalt und gesellschaftliche Teilhabe der ZHAW

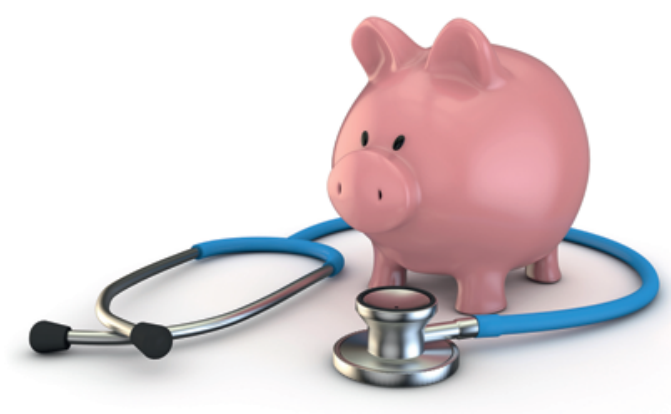

\begin{abstract}
Am 8. November 1982 fand auf Einladung von Bundesrat Hürlimann die Nationale Sparkonferenz im Gesundheitswesen statt. Seither ist die Kostenexplosion ein Dauerthema. Angesichts der weiterhin steigenden Gesundheitsausgaben liegt der Schluss nahe, alle Diskussionen und Massnahmen seien wirkungslos geblieben. Dies wäre allerdings eine Fehleinschätzung. ${ }^{1}$
\end{abstract}

\footnotetext{
1 Dieser Beitrag fasst kurz die wichtigsten Ergebnisse einer auf Dokumentenanalyse und Interviews mit 14 Protagonisten des Gesundheitswesens beruhenden Studie zusammen. Ein längerer Forschungsbericht kann auf der Website der Schweizerischen Ärztezeitung heruntergeladen werden.
}

Der jahrzehntelange Fokus auf Kosten und Effizienz hat dazu geführt, dass sich im schweizerischen Gesundheitswesen eine damals ganz neue Sichtweise fest- und durchgesetzt hat: der ökonomische Blick. Hinter der Fassade der politischen Ränkespiele und Blockaden haben sich weitreichende Umbrüche vollzogen, die sich als Ökonomisierung beschreiben lassen. Im Namen der Kosteneindämmung wird nicht das Ausgabenwachstum gebremst, sondern das Gesundheitswesen in einen Markt verwandelt. Es gibt allerdings keinen Grund, zu glauben, der Markt führe zu tieferen Gesundheitsausgaben. Internationale Vergleiche legen den gegenteiligen Schluss nahe, mit den USA als prominentes Beispiel für ein System mit Marktmechanismen und rekordhohen Ausgaben.

\section{Résumé}

Depuis 35 ans, le débat sur l'explosion des coûts domine la politique de la santé publique en Suisse. Etant donné que les coûts continuent d'augmenter, on pourrait penser que tout ce débat n'a eu aucun effet. Cela n'est pas vrai. L'étude dont les résultats sont présentés dans cet article montre que l'attention portée aux coûts a permis au regard économique de s'installer au sein du système de santé. Nous y sommes habitués aujourd'hui, mais cela n'allait pas de soi à l'époque. Sous l'effet d'un tribunal économique permanent (Foucault), le système de santé se transforme de plus en plus en marché. La tendance à la marchandisation est illustrée dans le domaine de l'expertise scientifique, des caisses maladie et du secteur hospitalier. II s'avère que marché et régulation politique ne sont pas antinomiques, mais vont main dans la main: I'introduction de l'assurance-maladie obligatoire en 1996 était un pas décisif dans cette direction. Finalement la question se pose de savoir si les cabinets médicaux finiront aussi par se transformer en entreprises, et ce que cela signifierait pour les médecins aussi bien que pour les patients.

\section{Gesundheitsökonomie}

Eine erste weitreichende Veränderung hat im Feld der wissenschaftlichen Expertise stattgefunden. Die alleinige Deutungshoheit der Mediziner war im Gesundheitswesen lange Zeit unbestritten. Auf staatlicher Seite waren Juristen für die Krankenversicherung zuständig, die nicht ins Metier der Ärzte eingriffen. Heute dagegen sehen sich alle Leistungserbringer vor ein permanentes «ökonomisches Tribunal» [1] gestellt. Die ärztlichen Tätigkeiten werden einer Wirtschaftlichkeitsprüfung unterzogen. Mit der Ökonomisierung sind neue Expertenfiguren entstanden. Etwa der Medizincontroller, der in Spitälern und Gesundheitsdiensten über die Kostenwahrheit wacht. Oder der Gesundheitsökonom, der als Politikberater dem Juristen den Rang abläuft. Dass das Bundesamt für Gesundheit seit 2010 erstmals überhaupt in dessen über hundertjähriger Geschichte nicht von einem Mediziner geleitet wird, passt ins Bild der Infragestellung ärztlicher Deutungshoheit.

Der zunehmende Einfluss ökonomischer Expertisen hat sich im Rahmen eines Reformdiskurses entfaltet, in dem es nicht nur um Kosteneinsparungen ging, sondern auch um Patientenrechte, Versorgungsqualität, Besserstellung der Pflege u.a. In der Schweiz wurde die 1976 durch Gerhard Kocher gegründete Gesellschaft für Gesundheitspolitik (SGGP) zum wichtigsten Forum, in dem sich die Reformer trafen. Dass man sich in der Gesundheitspolitik für die Meinung von Ökonomen interessierte, war damals ebenso neu wie die Tatsache, dass sich einige Ökonomen für das Gesundheitswesen zu interessieren begannen. Zu deren ersten Vertretern zählten Heinz Locher im Kanton Bern und Gianfranco Domenighetti im Tessin sowie die Universitätsprofessoren Robert Leu (Bern), Jürg Sommer (Basel) und Peter 
Zweifel (Zürich). Als einer der wenigen Mediziner, die den Reformdiskurs aktiv mitgestalteten, war Felix Gutzwiller durch den Besuch eines Master of Public Health in Harvard mit gesundheitsökonomischen Fragen vertraut geworden. Zurück in der Schweiz, leitete er das Nationalfondsprogramm "Wirtschaftlichkeit und Wirksamkeit im Gesundheitswesen» [2]

\section{Kassensterben}

Kein Stein ist bei den Krankenkassen auf dem anderen stehen geblieben. Von den über tausend Kassen der 1960er Jahre sind noch etwa 50 übrig. Dieser dramatische Konzentrationsprozess war zugleich eine regelrechte Verwandlung: Meistens sind an die Stelle von Vereinen, Stiftungen und Genossenschaften als Versicherungsträger private Versicherungsgesellschaften getreten.

Wie kaum eine andere Person verkörperte Manfred Manser diesen Prozess: Nach der Lehre bei einer kleinen örtlichen Kasse stieg er bei der Helvetia Stufe für Stufe bis nach ganz oben auf, wurde Mitte der 1990er Jahre zum Architekten der Fusion mit Artisana zur Helsana und agierte als deren Direktor bis zur Pensionierung im Jahr 2010. Aus dem Verein «Helvetia» mit lokalen Sektionen und Delegiertenversammlungen wurde die Helsana AG mit einer Holdingstruktur, die an die Anforderungen des neuen Krankenversicherungsgesetzes von 1994 angepasst war. Manser kann sich noch an ehrenamtliche Kassenfunktionäre erinnern, die ihre Versicherten zu Hause in der Stube empfingen, sowie an Kunden, die an den HelvetiaSchalter kamen, um zu plaudern, nicht um Versicherungsgeschäfte zu tätigen. ${ }^{2}$ Sein Nachfolger, Helsana-CEO Daniel H. Schmutz, der zuvor bei Boston Consulting Group und Swiss Life tätig gewesen war, kennt solche Geschichten höchstens vom Hörensagen. So steht diese Personalie beispielhaft für den Übergang von einem Geschäftsmodell, das noch durch und durch von den Besonderheiten der Krankenversicherung geprägt war, hin zu einer Vorstellung, der zu Folge es sich um ein Versicherungsgeschäft wie ein anderes handelt.

\section{Spitalmanagement}

Interview mit Manfred Manser vom 14. März 2016. 3 Bundesamt für Statistik, Krankenhausstatistik, T14: https://www.bfs.admin. $\mathrm{ch} / \mathrm{bfs} / \mathrm{de} / \mathrm{home} /$ statistiken/gesundheit/ erhebungen/ks.html

4 Interview mit Rita Ziegle vom 15. Juni 2016 dauer lassen sich als Indikatoren der Produktivitätssteigerung lesen. Einen Hinweis zur Ökonomisierung gibt auch die Veränderung der Rechtsformen. Laut einer Studie der Credit Suisse sind folgende Formen auf dem Rückzug: das Spital als Teil der öffentlichen Verwaltung, als Zweckverband, als öffentliche Stiftung oder als unselbständige öffentliche Anstalt; im Gegensatz dazu verzeichnen die Formen der Aktiengesellschaft und der selbständigen öffentlichen Anstalt einen markanten Zuwachs [3]. Hinzu kommt der Paradigmenwechsel in der Finanzierung: Waren die Spitalfinanzen traditionell Bestandteil der Kantons- oder Gemeindebudgets, wurde in den 1980er und 1990er Jahren auf Leistungsverträge mit Globalbudgets umgestellt, bevor sich ab der Jahrtausendwende Fallpauschalen durchsetzten, seit 2012 mit SwissDRG flächendeckend für sämtliche Akutspitäler in der Schweiz.

Rita Ziegler, Direktorin des UniversitätsSpitals Zürich von 2008 bis 2016, erinnert sich, wie noch Ende der 1980er Jahre in den Spitälern «nichts so lief, wie man es in der Betriebswirtschaft erwarten würde». Man habe nicht auf die Kosten geschaut, «man hat nicht gerechnet $»^{4}$. Der Kontrast zu heute ist markant. Es gab damals auch keine Spitalmanager, sondern bloss Spitaldirektoren, die eine eher symbolische Funktion innehatten. Die Chefärzte waren nicht dem Direktor unterstellt, sondern den politischen Verantwortungsträgern. Kaum einer verkörpert die neue Figur des Spitalmanagers und CEO so selbstbewusst wie Arnold Bachmann, der Direktor des Kantonsspitals Graubünden. Der Ökonom und ehemalige Manager von Ems Chemie zeichnet verantwortlich für die Fusion der drei Churer Spitäler zum Kantonsspital Graubünden. Das Spital ist für ihn längst nicht mehr eine sozial verwaltete und politisch kontrollierte Einrichtung, sondern ein Unternehmen, das auf dem Markt nach Wachstum strebt und dabei Allianzen eingeht, oder sich Konkurrenten einverleibt.

\section{Wettbewerb}

In der vorherrschenden Meinung stehen Markt und Staat im Gegensatz zueinander, und deshalb müsste die Ökonomisierung zu einem Rückzug des Staats führen. In der Wirklichkeit beobachten wir aber etwas anderes: eine zunehmende staatliche Regulierung des Gesundheitswesens. Aus wirtschaftssoziologischer Sicht $[4,5]$ ist das aber keineswegs überraschend: Der Markt ist nicht etwas, das von Natur aus, ohne Zutun des Staates entsteht. Wettbewerb ist ein Phänomen, das politisch erzeugt wird. In dieser Perspektive lässt sich die Einführung der obligatorischen Krankenversicherung in der Schweiz Mitte der 1990er Jahre als wich- 
tiger Schritt im Prozess der Ökonomisierung betrachten. Sie gab dem Staat neue Instrumente an die Hand, um den Wettbewerb im Gesundheitswesen sowohl zu fördern als auch zu gestalten.

Im Krankenversicherungsgesetz von 1994 kam der Glaube zum Ausdruck, die Herstellung von mehr Wettbewerb führe zu Kostensenkung. Zugleich führte das Gesetz transparentere Grundlagen des Wettbewerbs ein: die Trennung von Grundversicherung und Zusatzversicherung, das Verbot der Benachteiligung von Versicherten nach Alter und Geschlecht, den Risikoausgleich zwischen den Kassen. Entscheidend war zudem der Anstoss für die Aushandlung einheitlicher Tarifstrukturen im stationären wie ambulanten Bereich. Die Schweiz verfügt heute mit SwissDRG und TARMED (bei allen Unterschieden zwischen beiden Tarifen) über Tarifstrukturen, die systematische Vergleiche zwischen allen Leistungserbringern erlauben. Dies ist für die Transformation des Gesundheitswesens in einen Markt von zentraler Bedeutung. Wie das betriebliche Rechnungswesen [6] bilden auch diese Tarifsysteme nicht einfach wirtschaftliche Realitäten $a b$, sondern produzieren erst die ökonomischen Fakten: Konkrete ärztliche und pflegerische Tätigkeiten werden aus ihrem sozialen Zusammenhang gerissen und in die Sprache der Ökonomie übersetzt durch Umwandlung in schlichte Kennziffern. Der Tarif bestimmt das Mass der Werte, an denen sich der Wettbewerb orientiert.

\section{Zukunftsmusik}

Scott [7] hat die Veränderungen im US-amerikanischen Gesundheitswesen während der zweiten Hälfte des 20. Jahrhunderts als Abfolge dreier Logiken analysiert. Auf die professionelle Logik und die Vorherrschaft der Ärzte folgte in den 1960er Jahren die staatliche Logik mit ihrer Macht der Bürokraten, bevor sich ab den 1980er Jahren eine Logik des Marktes durchsetzte, welche die Macht in den Händen von Managern konzentriert. Im Vergleich zu den USA, wo der Staat durch die Gründung von Medicaid und Medicare aus dem Nichts zum grössten Einkäufer medizinischer Leistungen wurde, hat die staatliche Logik in der Schweiz nie derart eklatant ins System eingegriffen. Eher hat staatliche Regulierung bei uns schleichend und als Pendant zur Ökonomisierung des Gesundheitswesens an Bedeutung gewonnen.

Korrespondenz:

PD Dr. phil. Peter Streckeisen Institut für Vielfalt und gesellschaftliche Teilhabe der ZHAW

Pfingstweidstrasse 96

CH-8005 Zürich

Tel. 0589348617

peter.streckeisen[at]zhaw.ch
Ansätzen bereits vorhanden: Entweder gründen Ärzte eigene Unternehmen, oder sie arbeiten als Angestellte in integrierten Gesundheitsunternehmen. Bei beiden Varianten ersetzen Gruppenpraxen die traditionelle Einzelpraxis, die der unternehmerischen Perspektive mit Blick auf Wachstumsmöglichkeiten allzu enge Grenzen setzt. Die Ärztestatistik zeigt, dass bereits ein deutlicher Trend zu Gruppenpraxen existiert, vor allem unter Frauen und in der jüngeren Generation [8] In den meisten Fällen handelt es sich aber noch um Gruppenpraxen ohne Budgetverantwortung gegenüber den Versicherungsträgern.

Traditionell verstanden sich die Ärzte als Angehörige eines freien Berufs [9]. Freiberufler oder Unternehmer zu sein ist allerdings nicht genau dasselbe: Die einen achten auf ihre Freiheit in Berufsstellung und -ausübung und fühlen sich einer liberalen Ethik verpflichtet, während die anderen nach Wachstum und Gewinn streben und sich an ökonomischen Kalkülen ausrichten. In der Schweiz bremst zurzeit der Vertragszwang zwischen Krankenkassen und Ärzten noch die Ausbreitung des Praxis-Unternehmertums. Doch nachdem unter dem Druck der steigenden Prämien bereits über 60 Prozent der Versicherten in der Wahl ihrer Versicherungsmodelle freiwillig auf die freie Arztwahl verzichtet haben [10], könnte die politische Unterstützung für diese Regelung bald rapide abnehmen und deren Abschaffung einen weiteren Ökonomisierungsschub auslösen.

Bildnachweis

(c) Robertds | Dreamstime.com

Literatur

1 Foucault M. Geschichte der Gouvernementalität II. Die Geburt der Biopolitik. Frankfurt/M.: Suhrkamp; 2004. S. 342.

2 Sommer J, Gutzwiller F. Wirtschaftlichkeit und Wirksamkeit im schweizerischen Gesundheitswesen. Bern: Huber; 1986.

3 Keating G, Adler O. Gesundheitswesen Schweiz 2013. Der Spitalmarkt im Wandel. Zürich: Credit Suisse Group; 2013. S. 18-20.

4 Dobbin F (ed.). The Sociology of the Economy. New York: Russell Sage; 2004.

5 Fligstein N. The Architecture of Markets. An Economic Sociology of Twenty-First Century Capitalist Societies. Princeton: Princeton University Press; 2001.

6 Chiapello E. Die Konstruktion der Wirtschaft durch das Rechnungswesen. In: Diaz-Bone R, Krell G. (Hg.). Diskurs und Ökonomie. Wiesbaden: VS; 2009. S. 125-49.

7 Scott RW. Competing Logics in Healthcare: Professional, State, and Managerial. In: Dobbin, F (ed.). The Sociology of the Economy. New York: Russell Sage; 2004. pp. 267-87.

8 Hostettler S, Kraft E. FMH-Ärztestatistik 2015. Zuwanderung grundlegend für Versorgungssystem. Schweizerische Ärztezeitung. 2016;97(12-13):448-53.

9 Kocher G. Verbandseinfluss auf die Gesetzgebung. Ärzteverbindung, Krankenkassenverbände und die Teilrevision 1964 des Kranken- und Unfallversicherungsgesetzes. Bern: Dürrenmatt; 1967. S. 212-21.

10 Senn N, Ebert Tiaré S, Cohidon C. La médecine de famille en Suisse. Analyse et perspectives sur la base des indicateurs du programme SPAM. Neuchâtel: Observatoire Suisse de la Santé; 2016. S. 34. 